\title{
PLAN PROSPECTIVO Y ESTRATÉGICO DEL PROGRAMA DE ADMINISTRACIÓN DE EMPRESAS DE LA UNIVERSIDAD NACIONAL ABIERTA Y A DISTANCIA - UNAD AL AÑO 2019
}

\author{
Andrea del Pilar Barrera Ortegón' \\ Marilú Avendaño Avendaño ${ }^{2}$ \\ Carlos William Mera Rodríguez ${ }^{3}$
}

Recibido: 7 de junio de 2013

Aceptado: 12 de agosto de 2013

El futuro no se predice, sino se construye

Maurice Blondel

\section{Resumen}

Este artículo pretende presentar los resultados obtenidos en el proyecto de investigación titulado Plan prospectivo y estratégico del programa de administración de empresas de la Universidad Nacional Abierta y a Distancia -UNAD al año 2019, que fue desarrollado por el grupo de investigaciones y estudios prospectivos y estratégicos (GIEPE) ${ }^{4}$ y que tiene por objetivo construir el escenario apuesta del programa de administración de empresas de la Escuela de Ciencias Administrativas, Contables, Económicas y de Negocios - ECACEN, para lo cual se fundamentó teórica, conceptual y metodológicamente de la prospectiva estratégica y específicamente del «Modelo Básico» de la prospectiva estratégica. Por último se presentará el escenario apuesta el cual se denominó: Programa Integral y para lograrlo se formulo un plan prospectivo y estratégico con cinco grandes estrategias: Excelencia docente, Ciencia, tecnología e innovación, Investigación y desarrollo tecnológico, acreditación de alta calidad e internacionalización.

Palabras claves: prospectiva estratégica, modelo prospectivo, escenario, administración.

\footnotetext{
${ }^{1}$ Administradora de empresas,

2 Administradora de empresas,

${ }^{3}$ Ph.D (c) Economía, Magister en Educación, Especialista en Pedagogía y Docencia Universitaria, Economista, Director Prospecta Colombia Director Grupo de Investigaciones y Estudios Prospectivos y Estratégicos - GIEPE, Docente Universidad Nacional Abierta y a Distancia.

${ }^{4}$ Grupo de investigaciones categorizado por el Departamento Nacional de Ciencia, Tecnología e Innovación - Colciencias de Colombia.
} 


\section{PROSPECTIVE STUDY OF BUSINESS ADMINISTRATION PROGRAM OF THE NATIONAL UNIVERSITY OPEN AND DISTANCE - UNAD THE YEAR 2019}

\section{Abstract}

This paper aims to present the results of the research project entitled "Prospective Plan business administration program of the Universidad Nacional Abierta y a Distancia - UNAD to year 2019", which was developed by the research group and strategic prospective studies (GIEPE) which aims to build the stage bet business administration program at the School of management Sciences, Accounting, Economics and Business - ECACEN, for theoretical foundation which is conceptually and methodologically strategic foresight and specifically "Basic Model" of strategic foresight. Finally, we present the best scenario which I call "integral program" and to achieve it formulated a strategic plan with five prospective major strategies: Teaching Excellence, Science, Technology and Innovation, Research and technological development, accreditation of high quality and internationalization.

Keywords: Strategic prospective, Model prospective, Stage, Administration.

\section{Introducción}

La universidad del futuro no se construye sobre la coyuntura de las necesidades actuales, se piensa y se diseña hoy sobre la base de las necesidades futuras. Es aquí donde se empieza a construir la universidad desde cada uno de sus programas bandera. Es por esta razón que se desarrolló este plan prospectivo y estratégico del programa de administración de empresas de la Escuela de Ciencias Administrativas, Contables, Económicas y de Negocios, el cual se realizó a través del «Modelo Básico» de prospectiva estratégica adaptado al contexto colombiano y latinoamericano por Mójica (2008), con el objetivo de diseñar los diferentes escenarios en los que pueda encontrarse la administración de empresas en el futuro.

Este modelo se compone de las siguientes etapas: 1) Revisión de las fuentes secundarias y elaboración del estado del arte relacionado con el objeto de estudio; 2) Información de las fuentes primarias a través de una encuesta a expertos Delphi; 3) Identificación de los factores de cambio con la información arrojada en la aplicación del método Delphi; 4) Selección de variables estratégicas o clave utilizando el método Micmac ó análisis estructural; 5) Construir los escenarios posibles, probables, alternos y apuesta donde la herramienta seleccionada es el eje de Peter Schwartz; 6) Diseño de estrategias pertinentes para lograr el escenario apuesta. Los métodos antes mencionados se utilizan gracias al gran aporte realizado por Godet (2000), en su caja de herramientas y a la disponibilidad de cada uno de estos Software libre en la página Web de la LIPSOR de Francia.

Como resultado de esta investigación se pretende presentar el escenario apuesta denominado «Programa Integral» y la formulación del plan prospectivo y estratégico con diferentes estrategias que permitirán lograr este escenario.

Para dar respuesta a los objetivos planteados se formuló la siguiente pregunta ¿Qué estrategias se deben diseñar para construir el escenario apuesta del programa de administración de empresas de la UNAD, que permita que el 
egresado de nuestra Universidad sea de alta calidad, competitivo y reconocido en el mercado laboral nacional e internacional?

\section{Desarrollo}

Los antecedentes del programa de administración de empresas que es el tema fundamental de esta investigación se encuentra estrechamente ligado a la historia de la Universidad y la antigua Facultad hoy Escuela, el génesis del programa se ubica en el año de 1984 cuando se inician con los programas tecnológicos en las Gestiones: Comercial y de Negocios, Industrial, Agropecuaria, Empresas Asociativas y Organizaciones Comunitarias, Obras Civiles y Construcciones, y de Transportes, así mismo con el ánimo de contar con el proceso de formación de pregrado en el año de 1991 se inicia el programa de Administración de empresas a nivel profesional mediante acuerdo 141 del 2 de agosto, expedido por el ICFES.

En el año 1997 la transformación institucional comienza a ser factor esencial de desarrollo para la oferta académica y gestión de Programas, es así como con la Ley 396 del 5 de agosto de 1997, la Unidad Universitaria del Sur de Bogotá (UNISUR), cambia su denominación por Universidad Nacional Abierta y a Distancia (UNAD). Así mismo el 19 de agosto de 1998 mediante el acuerdo 023 del Consejo Directivo se crea la Especialización en Gerencia Estratégica de Mercadeo. El 7 de septiembre de 2005 se crea la Especialización en Gestión de Proyectos; El 24 de junio de 2008 mediante el acuerdo 002 se crea la Especialización en Gestión Pública. El 3 de Noviembre de 2009, mediante el acuerdo 011 del Consejo Superior Universitario se crea la Maestría en Administración de Organizaciones lo que ha permitido el fortalecimiento de la oferta posgradual de ECACEN.

En el año 2007, ECACEN toma la decisión de iniciar el proceso de autoevaluación con fines de acreditación para el Programa de Administración de Empresas, que, sumado a la evolución de la UNAD y de la hoy ECACEN, vendría a soportar una cadena de formación de gran impacto social, con identidad propia y fruto de la maduración y la experiencia. En el mes de julio de 2011 se radica ante el MEN los documentos solicitados por el mismo con el fin de acceder a la renovación del registro calificado del programa de Administración de Empresas cuyo resultado fue la obtención del Registro Calificado No 12929 de 10 de octubre de 2012 por una vigencia de siete (7) años.

Por lo anterior la ECACEN ya no sólo se dedica a la formación de Tecnólogos y Administradores de Empresas sino que aumentó su potencial con la posibilidad de ofertar Programas derivados de las disciplinas que se asocian en las áreas de conocimiento que originan su denominación, sino que también se implica en el aporte al desarrollo de nuevas cadenas de formación y Programas académicos de otras Escuelas de la UNAD.

De igual manera para desarrollar este ejercicio tan importante, vital y complejo para el futuro de la administración de empresas se tomo la decisión de utilizar una disciplina y sus diferentes herramientas como es la Prospectiva y es así como en primer lugar realizaremos una fundamentación y conceptualización de esta y del «Modelo Básico» y sus diferentes atapas.

La prospectiva parte del concepto que el futuro aún no existe y se puede concebir como una realizar múltiple Jouvenel, (1993) y que depende solamente de la acción del hombre Godet, (2000). Por esa razón, el hombre puede construir el mejor futuro posible, para lo cual debe tomar las decisiones correctas en el momento apropiado.

Jouvenel (1993) plantea que existen dos formas de ver el futuro, la primera como una realidad 
única, propia por los oráculos, profetas y adivinos. La segunda forma de ver el futuro es como una realidad múltiple, estos son los futuros posibles que Jouvenel denominó futuribles.

En el ámbito teórico, se destacan Gastón Berger y Bertrand y Hugues de Jouvenel como precursores de la prospectiva en Francia a mediados de los años sesenta y luego retomada por Michel Godet y en esta parte del mundo contamos con representantes como Francisco José Mójica, Eduardo Raúl Balbi, Tomas Miklos entre otros.

Berger (1967) uno de los primeros inspiradores de la prospectiva en Francia, define la prospectiva como la ciencia que estudia el futuro para comprenderlo y poder influir en él.

Balbi (2008) define la Prospectiva como la disciplina que ha conseguido convertirse en la herramienta clave de esa construcción del futuro deseado y posible, y en particular, sus últimos desarrollos que arriban a la llamada Prospectiva Estratégica constituyen el basamento fundamental del liderazgo y del management moderno.

La Prospectiva es una disciplina que nos permite construir múltiples escenarios a través de un ejercicio de participación y consenso entre los expertos y actores sociales relacionados con el objeto de estudio. Es por esta razón que se decidió utilizar estas herramientas para construir el escenario apuesta de la administración de empresas y específicamente seleccionamos el Modelo Básico de la Prospectiva Estratégica.

\section{Modelo básico de la prospectiva estratégica $^{5}$}

El «Modelo Básico» de prospectiva estratégica de la «escuela voluntarista» es una adaptación

5 El Modelo «Avanzado» de Prospectiva Estratégica se utiliza en situaciones que demandan mayor profundidad y con organizaciones que no están coartadas por el calendario es muy agradable tomarse su tiempo y servirse de la panoplia que contempla la «Caja de Herramientas» del maestro Godet. del profesor Francisco José Mójica ${ }^{6}$ (2008) a las condiciones propias del entorno colombiano y latinoamericano.

Grafico 1. Etapas fundamentales de la prospectiva estratégica

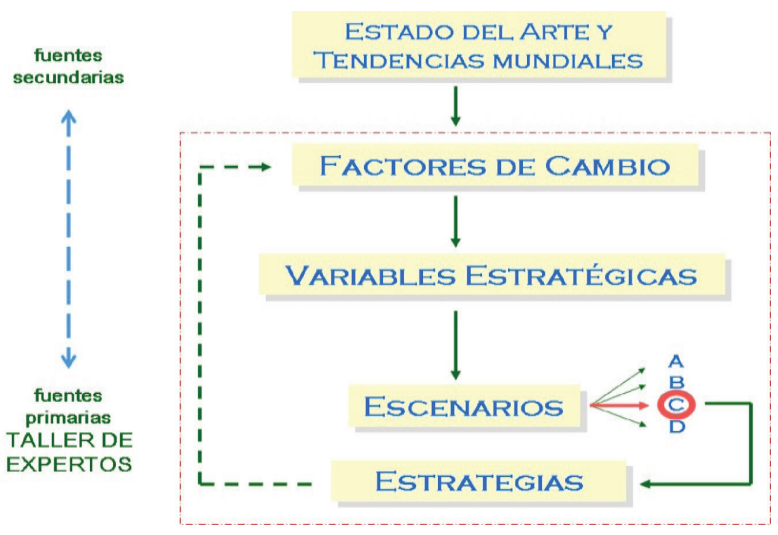

Fuente: Francisco José Mojica

Para una mejor comprensión del Modelo «Avanzado» de Prospectiva Estratégica, a continuación realizaremos una breve descripción de cada una de las fases que lo componen. (Como se puede ver en grafico No.1).

Fase 1: Revisión fuentes secundarias y elaboración del estado del arte: Se realiza un estado del arte que no es otra cosa que ir detrás de las huellas del objeto de estudio, en esta fase se estudia la situación actual del programa, nos preguntamos cómo es hoy el programa y también como era en el pasado y que estudios se han realizado sobre el futuro de la administración. De igual manera se realiza un estudio de vigilancia tecnológica e inteligencia competitiva que nos permita identificar las tendencias mundiales en lo educativo, tecnológico y las mejores prácticas, para el desarrollo de estas etapas se utilizaron bases de datos, buscadores de datos especializados, tanques de pensamiento, centros de investigación, centros de gestión de conocimiento especializado y observatorios.

\footnotetext{
6 Francisco José Mojica es Doctor en Ciencias Humanas de la Universidad de París V «René Descartes», estudió Prospectiva de la mano del profesor Michel Godet en el Lipsor de París, actualmente dirige el Doctorado en Ciencias de la Administración y el «Centro Pensamiento Estratégico y Prospectiva» de la Universidad Externado de Colombia.
} 
Fase 2: Información de las fuentes primarias: En esta etapa se diseña y aplica una encuesta Delphi, que según la Rand Corporación ${ }^{7}$ tiene como objetivo, partiendo de la consulta a expertos y gracias a cuestionarios sucesivos, poner de relieve convergencias de opinión y despejar ciertos consensos sobre temas precisos. El objetivo más frecuente de la encuesta Delphi es hacer llegar la luz que aportan determinados especialistas sobre las zonas de incertidumbre de determinado tema para tomar así mejores decisiones.

Fase 3: Identificación de factores de cambio: Los factores de cambio son las características de la organización que se expresan en fenómenos económicos, sociales, culturales, tecnológicos, políticos, ambientales, educativos, etc. En esta etapa se pretende con la ayuda de una encuesta Delphi y los expertos participantes, identificar cuáles son los factores de cambio que incidirán en el futuro de la administración. Para este plan prospectivo se selecciono como herramienta de apoyo la Matriz del Cambio de Godet $^{8}$ (2000), que nos permite identificar los cambios temidos, presentidos y anhelados en el futuro.

Fase 4: Selección de las variables estratégicas: Es importante tener en cuenta que no todos los factores encontrados en la etapa anterior son importantes, sino algunos que llamaremos variables estratégicas ó variables clave. Para la selección de estas utilizaremos el método Micmac ó «análisis estructural» creado por Godet (2000) y presentado en su aporte a la prospectiva en la «Caja de Herramientas»?.

La Rand Corporation fue la organización encargada de colocar a punto esta técnica con el apoyo de Olaf Helmer, Theodore J. Gordon y Norman C. Dalkey. Los primeros elementos de esta técnica los aportaron Dalkey y Helmer en 1953. Alcanzó su auge a mediados de los sesenta en el marco de los ejercicios de previsión tecnológica. La técnica Delphi original está presente en Analysis of the future: the Delphi methode, de Olaf Helmer (Rand Corporation, 1967, P-3558) y en The Delphi method: anexperimental study of group opinion de Norman C. Dalkey (Rand Corporation, 1969).

8 u uniz de

${ }^{9}$ Con el nombre de «Caja de Herramientas», Michel G odet nominó una de sus obras que

contiene las diferentes técnicas prospectivas producidas por él. Esta obra es electrónica y se puede descargar de la página web del autor: www.laprospective.com
Fase 5. Construcción de escenarios: Para la construcción de los escenarios se empleara la técnica de los «ejes de Schwartz» creado por Schwartz (1991), con la cual plantearemos los escenarios posibles, tendenciales, exploratorios y apuesta. En esta etapa no solo se diseñaran los escenarios, sino se realizará un análisis y explicación de cada uno de los escenarios, lo que nos permitirá la elección de un «escenario apuesta».

Fase 6: Diseño de estrategias: La última etapa del modelo es la definición de estrategias que se desarrollará a través de un plan prospectivo y estratégico planteado por Mera (2006), compuesto por los objetivos estratégicos, metas, actividades, acciones, instituciones que intervienes, responsables y planes de seguimiento.

\section{Resultados obtenidos a través del proceso prospectivo}

Después de presentar la metodología que se utilizo para el plan prospectivo y estratégico de la Administración del futuro y definir cada una de sus etapas fundamentales, se plantearon cuatro preguntas esenciales que complementan el modelo del profesor Mojica y posteriormente se desarrollo cada una de las etapas del modelo de la siguiente manera:

\section{Estado del Arte}

Actualmente, el programa se caracteriza por su enfoque y formación integral puesto que es altamente competitivo en los campos de conocimiento profesional, disciplinar, organizacional, investigativo, telemático, de idiomas y humanístico. Del total de 160 créditos académicos que posee, 120 de ellos son obligatorios y 40 son electivos. Del total de los créditos básicos, el $12 \%$ está compuesto por cursos del campo humanístico, el $25 \%$ está dedicado al campo investigativo y el $49 \%$ restante, está compuesto por el campo disciplinar y profesional, desatacándose la formación matemática, económica, 
solidaria, comunitaria, de producción y operaciones, logística, comercial, financiera y gerencial. Registro Calificado MEN №. 1679 Resolución №. 3959 del 18 de julio de 2006. SNIES : 210246580001100112300

Las visiones prospectivas del programa nos plantean que los medios de comunicación hablan continuamente de la expansión y retroceso de la Nueva Economía; del éxito y fracaso de las empresas virtuales; de los efectos de la Globalización y los movimientos sociales que manifiestan estar en su contra. $Y$ vemos como las facultades de administración, las administraciones públicas y las empresas como tal están requiriendo profesionales a la altura de estos cambios tan agiles. Todas estas visiones y conceptos tienen un componente común: El desarrollo de las Tecnologías de la Información y la Comunicación (TIC), y más concretamente de Internet y la World Wide Web (WWW), que hacen posible la interacción entre actores, en cualquier parte del mundo, en tiempo real y a bajo coste.
«En los últimos años distintas reflexiones y estudios consideran que una nueva etapa en la Historia de la Humanidad ha comenzado: la Era Informacional; que vivimos en un Mundo Digital y en una Sociedad Red y de la Información y el Conocimiento ubicada en el Tercer Entorno". Así pues, teniendo en cuenta el horizonte que se avecina se pretende reflexionar, de manera preliminar y desde un fundamento realista, sobre algunos de los problemas que deberá afrontar La Escuela de Ciencias Administrativas, Contables, económicas y de Negocios ECACEN, al darse cuenta de que debe anticiparse a conocer como es el Administrador de Empresas que requerirá el país tanto en el ámbito privado como público; de la misma manera el programa de administración debe identificar cual es el currículo, la estructura docente, la proyección de la carrera y los factores internos a corregir y así obtener los recursos físico y humanos adecuados para lograr estar preparado ante los cambios que se avecinan en los próximos 10 años.

\section{Preguntas portadoras de futuro.}

\begin{tabular}{|c|c|c|}
\hline VARIABLES & $\begin{array}{l}\text { ¿Cuáles son los aspectos clave } \\
\text { del tema que estamos estudiando? }\end{array}$ & ¿En dónde estamos? \\
\hline $\begin{array}{l}\text { PAPEL DE LOS ACTORES } \\
\text { SOCIALES }\end{array}$ & $\begin{array}{l}\text { ¿Cuál es el comportamiento de los } \\
\text { actores sociales? }\end{array}$ & $\begin{array}{l}\text { ¿Cómo están operando } \\
\text { los actores sociales? }\end{array}$ \\
\hline ESCENARIOS & ¿Qué puede pasar en el futuro? & $\begin{array}{l}\text { ¿Para dónde vamos? } \\
\text { ¿Hacia qué otros sitios } \\
\text { podemos encaminarnos? } \\
\text { ¿Cuál es nuestra opción } \\
\text { más conveniente? }\end{array}$ \\
\hline ESTRATEGIAS & $\begin{array}{l}\text { ¿Qué debemos hacer desde el } \\
\text { presente para construir nuestra } \\
\text { mejor opción de futuro? }\end{array}$ & $\begin{array}{l}\text { ¿Qué objetivos y metas debemos } \\
\text { alcanzar y a través de qué } \\
\text { acciones? }\end{array}$ \\
\hline
\end{tabular}

Fuente: Caja de herramientas - Michel Godet

\section{Expertos Participantes}

Los expertos que participaron en el ejercicio prospectivo fueron: Rector, Vicerrectores, Decanos Nacionales, Decanos Zonales, Secretarias Académicas, Coordinadora De Cadena De For- mación, Coordinadores Académico, Coordinador De Investigación, Coordinador De Concejeros, Asesores, Docentes, Tutores, Consejeros, Estudiantes, Egresados, Sector Productivo. 


\section{Método Micmac - Matriz de Impactos Cruzados - Identificación de factores de cambio:}

Para la selección de las variables claves se utilizó el Método Micmac ó análisis estructural creado por Godet (1990), que tiene por objetivo identificar las variables claves del ejercicio prospectivo. Las actividades desarrolladas en el análisis estructural permiten realizar un documento elaborado (informe) por el grupo que desarrolla el Plan Prospectivo del programa de Administración de Empresas para el 2019 y en el cual participaron los expertos invitados.

Las variables son fenómenos sobre los cuales no tenemos certeza con respecto a su evolución en el futuro, se pueden agrupar en categorías: económica, social, política, educativa, cultural, ambiental, científica, tecnológica, etc., para identificar estos factores de cambio se empleo la matriz DOFA y posteriormente el método Micmac para seleccionar las variables estratégicas ó claves.

\section{Identificación de factores de cambio - Método Mic Mac}

Los factores de cambio son las características de la organización que se expresan en fenómenos económicos, sociales, culturales, tecnológicos, políticos, ambientales, educativos, etc; a continuación se presentan los factores de cambio o variables del plan prospectivo y estratégico del programa de administración de empresas de la Universidad Nacional Abierta y a Distancia - UNAD al año 2019 que luego se calificaran de acuerdo a su influencia

\begin{tabular}{|c|c|c|c|}
\hline \multicolumn{4}{|c|}{ FACTORES DE CAMBIO Y/O VARIABLES } \\
\hline ITEM & NOMBRE LARGO & $\begin{array}{l}\text { NOMBRE } \\
\text { CORTO }\end{array}$ & DESCRIPCIÓN \\
\hline V1 & ACREDITACION ALTA CALIDAD & ACRD.ALT.C & $\begin{array}{l}\text { Los establecimientos educativos que se orientan a la } \\
\text { excelencia en la gestión pueden optar por procesos de } \\
\text { acreditación o certificación, que incorporan autoevaluación, } \\
\text { evaluación externa y procesos de mejoramiento. }\end{array}$ \\
\hline V2 & POLÍTICAS DE CALIFICACIÓN & POL.CALIF & $\begin{array}{l}\text { Creación de condiciones y la orientación de acciones de tipo } \\
\text { evaluativo en el proceso que tiene como finalidad determinar el } \\
\text { grado de eficacia y eficiencia, con que han sido empleados los } \\
\text { recursos destinados a alcanzar los objetivos previstos }\end{array}$ \\
\hline V3 & IMPACTO SOCIAL & IMPCT.SOSC & $\begin{array}{l}\text { Consecuencia directa sobre la población, originada por la facilidad } \\
\text { actual de obtener educación por medio de las tecnologías de } \\
\text { información }\end{array}$ \\
\hline V4 & TRABAJO EN RED & TRB.RED & $\begin{array}{l}\text { Grupo de actividades que pueden realizarse de forma simultánea } \\
\text { entre varias personas o grupos de éstas, empleando el ciberespacio } \\
\text { (Internet o redes internas) }\end{array}$ \\
\hline V5 & COMPETITIVIDAD & COMPE. & $\begin{array}{l}\text { Capacidad de generar la mayor satisfacción de los consumidores } \\
\text { fijado un precio o la capacidad de poder ofrecer un menor precio } \\
\text { fijada una cierta calidad, es decir, la optimización de la } \\
\text { satisfacción o el precio fijados algunos factores. }\end{array}$ \\
\hline v6 & ACOMPAÑAMIENTO TUTORIAL & ACOM.TUT. & $\begin{array}{l}\text { Direccionamiento de una asignatura, proyecto o investigación por } \\
\text { medio de una persona capacitada y experta en un campo específico }\end{array}$ \\
\hline v7 & $\begin{array}{l}\text { COBERTURA NACIONAL } \\
\text { ESTUDIANTES }\end{array}$ & COBR.NAL.E & $\begin{array}{l}\text { Proporción de alumnos atendidos en un nivel educativo con } \\
\text { respecto a la demanda. }\end{array}$ \\
\hline
\end{tabular}




\begin{tabular}{|c|c|c|c|}
\hline \multicolumn{4}{|c|}{ FACTORES DE CAMBIO Y/O VARIABLES } \\
\hline ITEM & NOMBRE LARGO & $\begin{array}{l}\text { NOMBRE } \\
\text { CORTO }\end{array}$ & DESCRIPCIÓN \\
\hline V8 & NORMATIVIDAD & NORMATIVID & $\begin{array}{l}\text { Estandares, parametros y normas establecidas para la realizacion } \\
\text { de un proyecto. Para el caso particular seria el conjunto de normas } \\
\text { que enmarcan el proceso de la educacion superior a distancia }\end{array}$ \\
\hline V9 & BILINGÚISMO & BILINGUISM & $\begin{array}{l}\text { Manejo y/o dominio de un segundo idioma diferente a la lengua } \\
\text { materna }\end{array}$ \\
\hline V10 & $\begin{array}{l}\text { OFERTA NACIONAL DE } \\
\text { PROGRAMAS }\end{array}$ & OFER.N.PRO & $\begin{array}{l}\text { Cantidad de programas educativos ofertados por una institución; } \\
\text { teniendo en cuenta un presupuesto y el manejo adecuado del los } \\
\text { recursos }\end{array}$ \\
\hline V11 & $\begin{array}{l}\text { PROGRAMAS DE BIENESTAR } \\
\text { UNIVERSITARIO }\end{array}$ & PROG.BIEN & $\begin{array}{l}\text { Conjunto de beneficios que una institución de educación superior } \\
\text { brinda a sus estudiantes, para garantizar unas condiciones básicas } \\
\text { de comodidad para el aprendizaje }\end{array}$ \\
\hline V12 & SERVICION AL CLIENTE & SERV.CLIT & $\begin{array}{l}\text { Grupo de personas encargadas de administrar las peticiones, } \\
\text { quejas y reclamos que presenta una población. Para el caso en } \\
\text { particular, se refiere a la comunidad interesada en los estudios de } \\
\text { educacion superior }\end{array}$ \\
\hline V13 & SISTEMAS DE COMUNICACIÓN & SIST.COM. & $\begin{array}{l}\text { Ayudas con las que cuenta una persona para comunicarse y o } \\
\text { relacionarse con otras para adquirir o compartir información de } \\
\text { diversos tipos }\end{array}$ \\
\hline V14 & AULAS VITRUALES & AU.VIR. & $\begin{array}{l}\text { Entornos de aprendizaje para facilitar a profesores la gestión de } \\
\text { cursos virtuales para sus estudiantes, utilizando las Tecnologías de } \\
\text { la Información y la comunicación }\end{array}$ \\
\hline V15 & $\begin{array}{l}\text { SISTEMA DE CALIDAD } \\
\text { ACADEMIA }\end{array}$ & SIST.CAL & $\begin{array}{l}\text { Referencia y el objetivo de cualquier actividad desarrollada en la } \\
\text { academia. }\end{array}$ \\
\hline V16 & MAPAS DE CONOCIMIENTO & MAP.CONCTO & $\begin{array}{l}\text { Diseños estructurados que nos permiten ampliar los contexto de } \\
\text { analisis, buscar ideas no usuales y tomar perspectivas distintas } \\
\text { para el conocimiento que hemos adquirido. }\end{array}$ \\
\hline V17 & $N^{\circ}$ ESTUDIANTES POR DOCENTE & $N^{\circ}$ ESTX & $\begin{array}{l}\text { El docente debe tener cierto número de estudiantes que permita la } \\
\text { realimentación de los trabajos de una manera idónea que permita } \\
\text { la construcción de conocimiento mediante la formación y el } \\
\text { aprendizaje }\end{array}$ \\
\hline V18 & $\begin{array}{c}\text { INVERSIÓN EN INVESTIGACIÓN } \\
\text { Y DESARROLLO } \\
\end{array}$ & INVR I\&D & $\begin{array}{l}\text { El dinero que se invierte para la obtención de nuevos conocimientos } \\
\text { y su aplicación para la solución a problemas }\end{array}$ \\
\hline V19 & MATERIAL DIDÁCTICO & MAT.DID. & Material alterno que facilita el proceso de enseñanza-aprendizaje \\
\hline V20 & $\begin{array}{l}\text { TECNOLOGIAS DE LA } \\
\text { INFORMACIÓN Y LA } \\
\text { COMUNICACIÓN }\end{array}$ & TICS & $\begin{array}{l}\text { Herramientas computacionales e informáticas que procesan, } \\
\text { almacenan, resumen, recuperan y presentan información } \\
\text { representada de la más variada forma. }\end{array}$ \\
\hline V21 & PROGRAMAS CERTIFICADOS & PROG.CERT. & $\begin{array}{l}\text { Programas educativos ofertados y que sean certificados por el } \\
\text { Ministerio de Educación }\end{array}$ \\
\hline V22 & NIVEL SALARIAL DEL DOCENTE & NIV.SALR.D & $\begin{array}{l}\text { Retribución a la función que cumple una persona experta en un } \\
\text { tema determinado y que centra su responsabilidad en el ambito } \\
\text { académico de docencia, investigación y proyección social }\end{array}$ \\
\hline V23 & $\begin{array}{l}\text { EXÁMENES DE CALIDAD DE LA } \\
\text { EDUCACION SUPERIOR }\end{array}$ & ECAES & $\begin{array}{l}\text { Prueba de conocimientos aplicada en Colombia a los estudiantes } \\
\text { que están en los últimos semestres de carreras profesionales. Son } \\
\text { un requisito para obtener el título profesional }\end{array}$ \\
\hline V24 & $\begin{array}{l}\text { INVERSIÓN EN } \\
\text { INTERNACIONALIZACIÓN }\end{array}$ & INV.INTERN & $\begin{array}{l}\text { Fomenta los lazos de cooperación e integración de las } \\
\text { Instituciones de Educación Superior con sus pares en otros } \\
\text { lugares del País, con el fin de alcanzar mayor presencia y } \\
\text { visibilidad en el país el cual es más globalizado. }\end{array}$ \\
\hline V25 & ALIANZAS ESTRATÉGICAS & ALZ. & $\begin{array}{l}\text { Es la asociación de dos o más personas físicas o jurídicas con el } \\
\text { objeto de generar -con los aportes de cada una de ellas- } \\
\text { proyectos de distinto tipo. }\end{array}$ \\
\hline
\end{tabular}

Fuente: Autores. 


\section{Selección de las variables estratégicas ó claves}

Para el plan prospectivo las variables a tener en cuenta son las "clave o estratégicas", que están ubicadas en la Zona de Conflicto, pero para indicar su selección está señalada por la diagonal de color rojo y específicamente en la parte superior derecha del plano de influencias dependencias directas. El análisis estructural nos permite ordenar el programa de administración de empresas como un sistema, es decir como un todo compuesto por elementos entre los cuales ocurren relaciones de interdependencia.

Grafica 1. Plano de influencias y/o dependencias directas

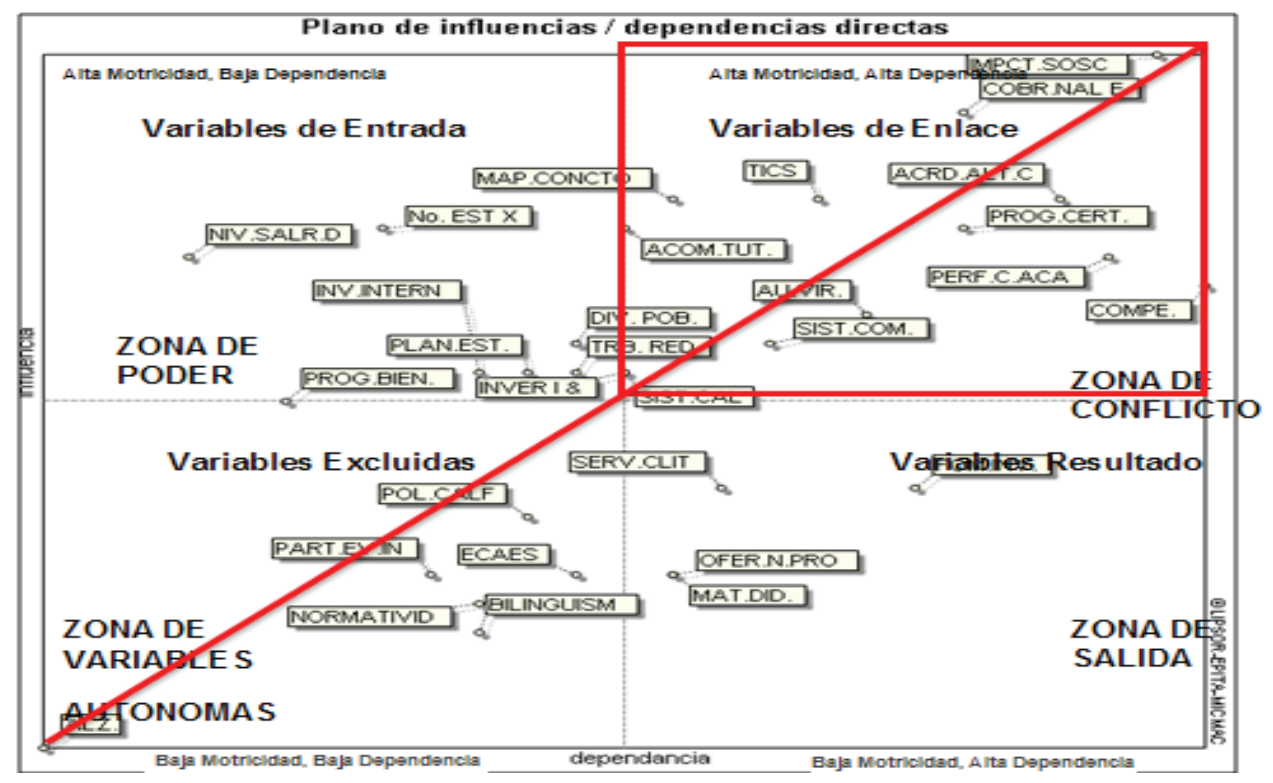

Fuente: Propia

\section{Análisis de Resultados}

En la zona de conflicto encontramos las variables de Alta Motricidad, Alta Dependencia, son las Variables Estratégicas o Variables Clave que seleccionamos para diseñar los diferentes escenarios. Desde el punto de vista depende de la zona de poder y a su vez con esta zona afectan a las variables de zona de salida, en prospectiva son denominados futuribles que a continuación presentamos:

- Programa Certificado con Alta Calidad (Los indicadores del esfuerzo incluyen la cantidad de títulos, diplomas, certificados; los cursos diseñados, elaborados e impartidos; el número de solicitudes, inscripciones, admisiones, exámenes, así como las creden- ciales otorgadas. El incremento en estas cantidades requiere de mayor capacidad para responder a las necesidades de los estudiantes)

- Perfil del Cuerpo Académico (Como actor educativo, centra su responsabilidad en el ámbito académico de docencia, investigación y proyección social. Desarrolla la planeación, diseño, seguimiento y actualización de currículos; elabora y actualiza materiales didácticos y objetos virtuales de aprendizaje; realiza el diseño de estrategias e indicadores de acompañamiento; asesora y hace seguimiento a estudiantes; diseña y elabora sistemas e instrumentos de evaluación de procesos de aprendizaje 
y bancos de pruebas; realiza diseño de estrategias para las interactividades)

- Acreditación de Alta Calidad: cumplir con el desarrollo de una cultura de calidad coherente con su modelo académico pedagógico, en sus diferentes ámbitos y multicontextos de actuación , trabajando responsablemente en el marco de su autonomía y misión universitaria con criterios innovadores que garantizan la satisfacción de los diferentes actores académicos, beneficiarios y usuarios de sus programas y servicios, así como la calidad, eficiencia y efectividad de su modelo de gestión y el mejoramiento continuo de sus procesos y recursos

- Cobertura a nivel nacional en todos los municipios colombianos e internacional en todos los puntos geográficos donde un estudiante requiera de nuestros servicios.

- Acompañamiento tutorial (En procesos de aprendizaje y la formación a distancia tiene un carácter pedagógico, razón por la cual es un elemento esencial de la Propuesta Pedagógica y contempla un acompañamiento, interactividad, asesorías académicas, orientaciones metodológicas, seguimiento a procesos de aprendizaje y evaluación de los procesos de aprendizaje)

- Sistemas de Comunicación (Ubicar al estudiante frente a los diferentes sistemas de transmisión de información, tanto en el marco tecnológico, como en su contexto socioeconómico a nivel nacional e internacional)
- Programas diseñados con fundamento en los mapas de conocimiento regionales, los núcleos problemicos de cada programa y las competencias que debe desarrollar cada estudiante nuestro para darle respuesta a las diferentes problemáticas del país, la región y las organizaciones.

- Aulas virtuales (El aula virtual no debe ser solo un mecanismo para la distribución de la información, sino que debe ser un sistema en donde las actividades involucradas en el proceso de aprendizaje puedan tomar lugar, es decir que debe permitir interactividad, comunicación, aplicación de los conocimientos, evaluación y manejo de la clase)

- Impacto Social (hace parte del subsistema de Dirección Estratégica; es una unidad misional que tiene como objeto la planeación, diseño, evaluación y proyección de las altas políticas institucionales para la creación de las condiciones reales de acompañamiento a los procesos de desarrollo comunitario y arraigo regional en la universidad)

- Fomento a la Investigación (Es la estrategia organizacional que asegura el cumplimiento de objetivos, la sustentabilidad de la investigación, la pertinencia regional, nacional y global, la visibilidad y la distribución de los productos y/o avances científicos. Como estrategia se compone del conjunto de procesos y elementos interrelacionados intencionalmente para el fomento y desarrollo del ejercicio investigativo en la UNAD) 
- $\quad$ TIC (El uso de las TIC representa una variación notable en la sociedad y a la larga un cambio en la educación, en las relaciones interpersonales y en la forma de difundir y generar conocimientos; Las tecnologías de la información y la comunicación son un conjunto de servicios, redes, software, aparatos que tienen como fin el mejoramiento de la calidad de vida de las personas dentro de un entorno, y que se integran a un sistema de información interconectado y complementario. Esta innovación servirá para romper las barreras que existen entre cada uno de ellos)

- Sistemas de calidad (son los que promueven el progreso de sus estudiantes en una amplia gama de logros intelectuales, sociales, morales y emocionales, teniendo en cuenta su nivel socioeconómico, su medio familiar y su aprendizaje previo. Un sistema escolar eficaz es el que maximiza la capacidad de las escuelas para alcanzar esos resultados)

- Mapas de conocimiento (tienen como objetivo servir de herramientas metodológicas, para la identificación de rutas pedagógicas y académicas, que den pistas para el diseño de programas educativos pertinentes y adaptados a poblaciones específicas en las regiones en las cuales la UNAD hace presencia)

\section{Método MACTOR - Método de actores o Matriz de Alianzas y Conflictos: Tácticas, Objetivos y Recomendaciones}

El método de análisis de juego de actores, MACTOR, según, Godet M, (1993) busca valorar las relaciones de fuerza entre los actores y estudiar sus convergencias y divergencias con respecto a un cierto número de posturas y de objetivos asociados. A partir de este análisis, el objetivo de la utilización del método MACTOR es el de facilitar a un actor una ayuda para la decisión de la puesta en marcha de su política de alianzas y de conflictos. El método MACTOR se enfoca fundamentalmente en la determinación de las motivaciones, conflictos y posibles alianzas estratégicas entre los Actores de cara al futuro.

En este método se requiere diligenciar dos cuadros de datos a partir de los cuales se obtienen múltiples páginas de listados de resultados y de esquemas.; es por esto que todo depende de la calidad de los datos de entrada así como de la capacidad de clasificar los resultados más pertinentes.

De esta forma, el análisis del juego de actores, la confrontación de sus proyectos, el examen de sus valoraciones de fuerzas (obstáculos y medios de acción) son esenciales a la hora de evaluar los retos estratégicos y las cuestiones clave para el futuro (resultados y consecuencias de los conflictos previsibles). 


\section{Identificación de Actores y sus objetivos - Método MACTOR}

\begin{tabular}{|c|c|c|c|c|}
\hline ITEM & ACTOR & $\begin{array}{l}\text { NOMBRE } \\
\text { CORTO }\end{array}$ & DESCRIPCIÓN & OBJETIVO \\
\hline $\mathrm{A}_{1}$ & $\begin{array}{c}\text { Ministerio de Educación } \\
\text { Nacional }\end{array}$ & MEN & $\begin{array}{l}\text { Institución adscripta al gobierno nacional que tiene como función formular la } \\
\text { política nacional de educación, regular y establecer los criterios y parámetros } \\
\text { técnicos cualitativos que contribuyan al mejoramiento del acceso, calidad y equidad } \\
\text { de la educación, en la atención integral a la primera infancia y en todos sus niveles y } \\
\text { modalidades. }\end{array}$ & $\begin{array}{l}\text { Formular Programas Nacionales De Apoyo a las } \\
\text { instituciones de educación superior a distancia }\end{array}$ \\
\hline $\mathrm{A}_{2}$ & $\begin{array}{c}\text { Instituto Colombiano para } \\
\text { el Fomento de la } \\
\text { Educación Superior }\end{array}$ & ICFES & $\begin{array}{l}\text { Institución adscripta al Ministerio de Educación Nacional que tiene como función el } \\
\text { Instituto gestiona y desarrolla investigación sobre la calidad de la educación }\end{array}$ & $\begin{array}{l}\text { Investigar Las Necesidades Y Evolución De La } \\
\text { Educación A Distancia }\end{array}$ \\
\hline $\mathrm{A}_{3}$ & \begin{tabular}{|c|} 
Instituto de Crédito \\
Educativo y Estudios en el \\
Exterior.
\end{tabular} & ICETEX & $\begin{array}{l}\text { Entidad dedicada a promover la Educación Superior a través del otorgamiento de } \\
\text { créditos educativos y su recaudo. }\end{array}$ & $\begin{array}{l}\text { Facilitar El Crédito Educativo Para Programas De } \\
\text { Formación A Distancia }\end{array}$ \\
\hline $\mathrm{A}_{4}$ & $\begin{array}{l}\text { Ministerio de las } \\
\text { Tecnologías de } \\
\text { Información y } \\
\text { Comunicación } \\
\end{array}$ & MINTIC & $\begin{array}{l}\text { Planea, regula y controla todos los servicios del sector de comunicaciones, los } \\
\text { servicios informáticos y de telemática, los servicios de valor agregado y los } \\
\text { servicios postales. }\end{array}$ & $\begin{array}{l}\text { Patrocinar La Utilización De Las Tic En Procesos } \\
\text { De Formación Profesional }\end{array}$ \\
\hline $\mathrm{A}_{5}$ & Rectoria & RECTORIA & $\begin{array}{l}\text { Es el alto gobierno de la Universidad y está dirigida por el Rector como } \\
\text { representante legal y primera autoridad ejecutiva. }\end{array}$ & $\begin{array}{l}\text { Dirigir y coordinar la gestión universitaria en todas } \\
\text { sus dimensiones organizacionales y ámbitos de } \\
\text { actuación. }\end{array}$ \\
\hline $\mathrm{A}_{6}$ & Sector Productivo & SECPROD & $\begin{array}{l}\text { Se refiere a las empresas del sector privado o público que están en el entorno de } \\
\text { las instituciones de educación superior a distancia. }\end{array}$ & $\begin{array}{l}\text { Proporcionar A Los Egresados De Educación A } \\
\text { Distancia, La Posibilidad De Acceso A Empleo }\end{array}$ \\
\hline $\mathrm{A}_{7}$ & $\begin{array}{l}\text { Consejo Nacional de } \\
\text { Acreditación }\end{array}$ & CNA & $\begin{array}{l}\text { Promueve y ejecuta la política de acreditación adoptada por el consejo nacional de } \\
\text { educación superior. }\end{array}$ & $\begin{array}{l}\text { Evaluar Y Acreditar Programas Ofertados Por } \\
\text { Modalidad A Distancia Que Cumplan Requisitos }\end{array}$ \\
\hline $\mathrm{A}_{8}$ & Comunidad Estudiantil & ESTUEAD & $\begin{array}{l}\text { Se refiere a los estudiantes que conforman el grupo poblacional que reciben } \\
\text { formación mediante modalidad a distancia. }\end{array}$ & $\begin{array}{l}\text { Compenetrarse Con La Metodología Educativa } \\
\text { Por La Cual Están Recibiendo Formación }\end{array}$ \\
\hline $\mathrm{A}_{9}$ & $\begin{array}{l}\text { Comité Nacional de } \\
\text { Investigación }\end{array}$ & COMNALINV & $\begin{array}{l}\text { organismo dedicado a la planeación, organización, y evaluación de actividades de } \\
\text { fomento, apoyo y coordinación de la investigación en la Universidad }\end{array}$ & $\begin{array}{l}\text { Ejecutar directamente las politicas y planes para la } \\
\text { gestión, consolidación y sostenimiento de la } \\
\text { investigacióno de la universidad }\end{array}$ \\
\hline $\mathrm{A}_{10}$ & Grupos de Investigación & GINVEAD & $\begin{array}{l}\text { Conjunto de personas que se reúnen para realizar investigación en una temática } \\
\text { dada, formulan uno o varios problemas de su interés, trazan un plan estratégico de } \\
\text { largo o mediano plazo para trabajar en él y producir unos resultados de } \\
\text { conocimiento sobre el tema cuestión. Para el caso son grupos de investigación } \\
\text { pertenecientes exclusivamente instituciones que ofertan programas de modalidad a } \\
\text { distancia. }\end{array}$ & $\begin{array}{l}\text { Fomentar la Investigaciónpara la generación de } \\
\text { conocimiento científico y de desarrollo } \\
\text { tecnológico, conformada por un equipo de } \\
\text { investigadores de varias disciplinas para el } \\
\text { desarrollo de proyectos. }\end{array}$ \\
\hline$A_{11}$ & $\begin{array}{c}\text { Vicerrectoria Académica } \\
\text { y de Investigación }\end{array}$ & VIACADIN & $\begin{array}{l}\text { Unidad estratégica responsable de dirigir políticas y acciones que permitan } \\
\text { consolidar el desarrollo académico, pedagógico e investigativo de la UNAD }\end{array}$ & $\begin{array}{c}\text { Articular el ejercicio académico, pedagógico e } \\
\text { investigativo a través de las Escuelas y de las } \\
\text { demás formas de organización que para tales fines } \\
\text { establezca la Rectoría. }\end{array}$ \\
\hline $\mathrm{A}_{12}$ & $\begin{array}{c}\text { Vicerrectoria de Medios y } \\
\text { Mediaciones }\end{array}$ & VIMMED & $\begin{array}{l}\text { Unidad encargada de los medios y mediaciones de la universidad mediante las } \\
\text { Tecnologías de la Información y Comunicación y la Web, que permite dinamizar } \\
\text { procesos de aprendizaje autónomo y colaborativo y la investigación. }\end{array}$ & $\begin{array}{l}\text { Proporcionar escenarios para la oferta de cursos } \\
\text { Web, objetos virtuales de aprendizaje, } \\
\text { simuladores y repositorios digitales. }\end{array}$ \\
\hline $\mathrm{A}_{13}$ & Consejo de Escuela & CONSESCU & Es el máximo órgano de gobierno de la Escuela & $\begin{array}{c}\text { Proponer la creación,modificación o supresión de } \\
\text { programas curriculares, de acuerdo con el } \\
\text { presente Estatuto y sus reglamentos. }\end{array}$ \\
\hline $\mathrm{A}_{14}$ & $\begin{array}{l}\text { Sistema Nacional de } \\
\text { Registro y Control }\end{array}$ & REGYCONTR & $\begin{array}{l}\text { Unidad encargada de proporcionar a los aspirantes la información correspondiente } \\
\text { a los requisitos para el ingreso a los programas académicos de la UNAD }\end{array}$ & $\begin{array}{c}\text { Registrar la información académica, legalizando la } \\
\text { matrícula del estudiante y el derecho al uso de los } \\
\text { servicios académicos de la Institución }\end{array}$ \\
\hline $\mathrm{A}_{15}$ & $\begin{array}{l}\text { Docentes, tutores o } \\
\text { instructores }\end{array}$ & DOCETUT & $\begin{array}{l}\text { El equipo docente sin importar su denominación, que ejerce tareas de formación } \\
\text { para el aprendizaje autónomo y actividades tutoriales en cada una de las } \\
\text { instituciones de educación superior que ofrece programas a distancia. }\end{array}$ & $\begin{array}{c}\text { Ayudar Al Estudiante de educación superior a } \\
\text { distancia para aprender a aprehender }\end{array}$ \\
\hline $\mathrm{A}_{16}$ & \begin{tabular}{c|} 
Departamento \\
Administrativo de Ciencia \\
Tecnología e Innovación
\end{tabular} & COLCIENC & $\begin{array}{l}\text { Entidad de orden nacional con autonomía administrativa y patrimonio independiente } \\
\text { adscripto al DANE. Su tarea consiste en planear, articular y apoyar el desarrollo } \\
\text { científico y tecnológico. }\end{array}$ & $\begin{array}{c}\text { Promover La Investigación en las instituciones de } \\
\text { educación a distancia }\end{array}$ \\
\hline $\mathrm{A}_{17}$ & Egresados & EGRESADO & $\begin{array}{l}\text { Se trata de aquellos profesionales que cursaron sus estudios en instituciones que } \\
\text { ofrecen educación a distancia, ya sean técnicos, tecnológicos, profesionales o } \\
\text { postgraduales. }\end{array}$ & $\begin{array}{l}\text { Aplicar Y Gestionar El Conocimiento en } \\
\text { interactuación con el sector productivo }\end{array}$ \\
\hline
\end{tabular}

Fuente: Propia 
Al procesar la información en el método MACTOR calificando la influencia de los actores entre sí y de igual manera la influencia de los diferentes actores con los objetivos de los actores podemos visualizar resultados como el siguiente:

Plano de influencias-dependencias entre actores.

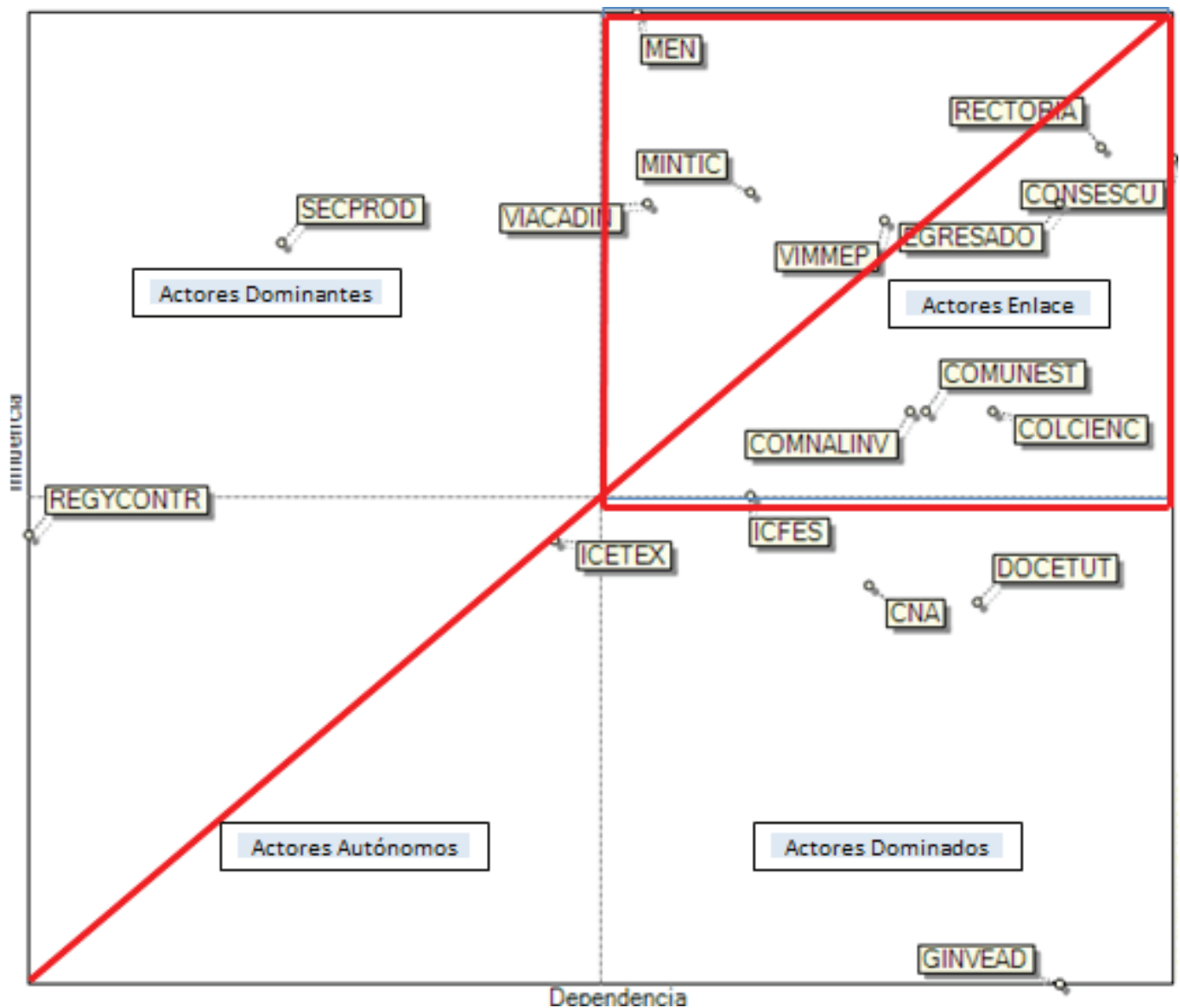

Fuente: Propia

El plano de Influencias y dependencias entre actores muestra de forma explícita el posicionamiento de los actores en el sistema, permite evidenciar la debilidad de los grupos de investigación de las instituciones de educación a distancia y el grado de poder del sector productivo que aunque no es el más influyente, es poco dependiente lo que lo destaca como un actor clave en el manejo de las variables. Se concluye que el programa de administración de empresas de la UNAD es un sistema altamente equilibrado donde la mayoría de actores son influyentes y dependientes a la vez, por tanto las acciones que hagan determina- da institución, organización o ente impactaran sobre los otros y viceversa.

Dentro de los actores de enlace se encuentran el Ministerio de Educación Nacional, Ministerio de las Tecnologías de la Información y la Comunicación, la rectoria, Consejo de Escuela, la comunidad estudiantil; Vicerrectoría Académica y de Investigación; Departamento Administrativo de Ciencia, Tecnología e innovación; Vicerrectoría de medios y mediaciones, egresados. Todos los anteriores son actores con considerable nivel de dependencia y de influencia; la existencia, misión, proyectos y programas que 
de ellos se deriven tienen un alto impacto sobre el sistema en general. Estos actores son los que le pueden brindar un mayor apoyo al programa de administración de empresas en la parte financiera, tecnológica y académica para que sea pertinente y acorde con las necesidades a nivel nacional.

- El Consejo de escuela cumple un papel preponderante en la definición de nuevas políticas para el mejoramiento de la educación dentro de la UNAD, ya que su papel es avalar y gestionar los proyectos en procura de este objetivo. En este sentido las recomendaciones y disposiciones emanadas por este actor tiene una influencia alta.

- La Vicerrectoria académica y de investigación involucrada con responsable de dirigir políticas y acciones que permitan consolidar el desarrollo académico, pedagógico e investigativo de la UNAD, logra tener una influencia alta sobre actores como la comunidad estudiantil, pero no logra impactar fuertemente en el sector privado.

- La comunidad Estudiantil representada en la necesidad de obtener una mejor educación frente a la competitividad del mercado laboral tanto privado como en el sector público, es un actor con dependencia y su influencia es entendida e un poco baja, porque no se le ha dado la importancia que se merece; además no hay grupos conformados que participen o exijan mejorar los medios y mediaciones para mejorar la educación en Colombia y la pertinencia de los programas académicos ofertados.

- Los grupos de Investigación como el Grupo de Investigación y Estudios Prospectivos y Estratégicos - GIEPE como actor importante debería tener un papel más protagónico, pero en realidad no lo cumple, puesto que los resultados y hallazgos que reportan en estudios, las universidades y los centros de investigación advirtiendo nuevos desarrollos o mejoras, no son tenidos en cuenta, bien sea, porque no son transferidos a los actores claves en la toma de decisiones sobre la educación en el país, o porque no son escuchados ya que se anteponen a estos, otros intereses.

\section{Escenarios futuros}

Los Escenarios Futuros procuran articular el pensamiento colectivo hacia el mañana, Implican imaginar, hacia adelante cómo será ese porvenir, generan el desafío de construir un camino hacia el futuro deseado; esta metodología puede aplicarse en el marco de una organización, sector o comunidad.

Es un proceso estructurado de exploración y reflexión, en el que los participantes construyen un entendimiento compartido y las acciones necesarias para alcanzar un futuro mejor, donde se identifica lo que ha ocurrido, está ocurriendo y podría ocurrir y lo que debería hacerse para lograr el resultado deseado, de esta manera se proyectan una gama de posibles resultados y permiten que las personas piensen acerca del futuro de diversas maneras; por lo tanto los participantes deberán: interpretar el pasado, entender el presente, identificar lo predecible y lo incierto, reconocer caminos factibles hacia el futuro y considerar interpretaciones divergentes.

El objetivo principal de los escenarios es identificar el mayor número posible de incertidumbres que afectan las decisiones estratégicas para las organizaciones, nos permiten ver cómo las decisiones que hoy se toman pueden jugar un papel evaluado y probado contra la incertidumbre del futuro. Según Mojica (2001). La construcción de escenarios consta de las siguientes etapas:

Teniendo en cuenta los resultados de los software MICMAC y MACTOR, que suministran 
información básica para realizar el análisis de un tema con orientación prospectiva, se siguen estas pautas:

a. Seleccione el tema que va analizar prospectivamente (Taller lluvia de Ideas)

b. Determine y profundice las variables más importantes (reciben el nombre de «variables clave y/o estratégicas») que conforman y definen el tema (Plano de influencias dependencias directas - Método MICMAC). Estas variables suelen pertenecer a categorías como las siguientes: 1. Económica 2. cultural 3. científico-tecnológica 4. Ambiental 5. Político-administrativa 6. Normativo-jurídica 7. Otras.

Se agrupan en dos categorías las «variables estratégicas»; para nuestro caso son las Educativas y tecnológicas (Tecnológico - Educativas) en la primera categoría y las Sociales y Políticas (Socio- Políticas) en la segunda categoría.

1. Asuma conjeturalmente que dentro del tiempo determinado (3 a 20 años) estas variables se irán a encontrar en una situación mejor o peor de la situación actual. Utilice las dos categorías o seleccione la variable más representativa de cada categoría. 2. Señale el mejoramiento de la situación con el signo +3 . Señale el empeoramiento de la situación con el signo - 4. No existen sino cuatro combinaciones posibles de los signos $+y$ -, por lo tanto, dentro de 3 - 20 años, las variables escogidas por usted deberán encontrarse en una de estas cuatro situaciones: (+ -) (-+) (--) (++)

c. Identifique los «actores enlace» que intervienen en el comportamiento del tema que está estudiado (Plano de influencias dependencias entre actores - Método MACTOR).

d. Indique el grado de poder, las alianzas y conflictos que ocurren entre ellos en relación con las variables anteriormente identificadas. Analice estas situaciones sirviéndose del grado de poder que maneja cada uno (Plano y/o gráfico de convergencias divergencias de objetivos entre actores o plano de distancias netas entre actores - Método MACTOR).

e. Retome nuevamente las «variables clave y/o estratégicas», verifique las tendencias que se dan en cada una de ellas y con esta información diseñe la imagen de lo que podría pasar en el tiempo determinado para su plan prospectivo (3-20 años), si continúan estas tendencias.

Para diseñar los escenarios se realizan conjeturas y se realizan preguntas como ¿qué condiciones tuvieron que haberse dado para que ocurriese cada uno de ellos?; se tienen en cuenta los conflictos y alianzas de los «actores sociales».

\section{Eje de peter schwartz: escenarios del} programa de administracion de empresas - unad 2019

Este es un método cualitativo que nos permite realizar diferentes «simulaciones de escenarios", definiendo posibles situaciones futuras para que nos indiquen las acciones estratégicas a desarrollar que nos permitan la consecución del escenario apuesta; de igual manera que contribuya a determinar como será el entorno competitivo en el medio y largo plazo (con distintos escenarios).

Los futuros posibles se pueden reconocer por medio de los «Ejes de Peter Schwartz» que permiten reducir las variables estratégicas a dos direccionadores de futuro; es decir pueden encontrarse en el futuro en su mejor o peor momento y además permiten reconocer que cada situación depende de lo que hagamos o dejemos de hacer en el presente. 
Eje de Peter Schwartz

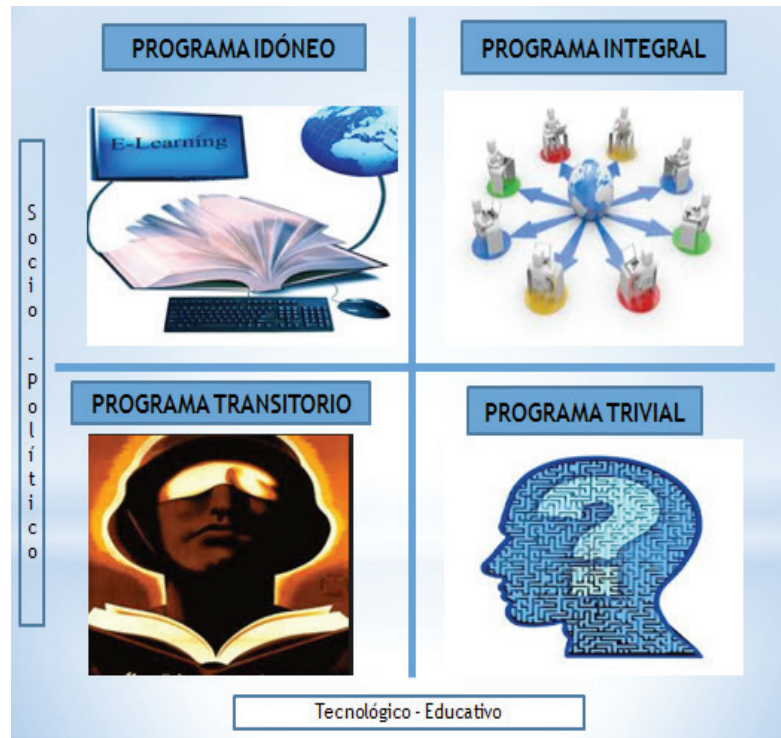

Fuente: Los Autores.

\section{Escenario Apuesta: Programa Integral.}

El planteamiento de este escenario, supone asumir el programa de administración de empresas como un concepto integrador que requiere:

Las universidad Nacional Abierta y a Distancia - UNAD aprovechará su programa de administración de empresas en la modalidad a distancia, que puede ser potencializado a través de las tecnologías de la información y la comunicación TIC, facilitando una interacción sinérgica a nivel interinstitucional por medio de convenios o alianzas estratégicas, que permitirán el intercambio sistémico de conocimientos, experiencias, propuestas y proyectos con otras regiones del país e incluso a nivel internacional y global.

El programa permite incentivar la actividad investigativa si se desarrollan proyectos que permitan vinculara estudiantes de diferentes regiones, donde las empresas como la sociedad aprovechan en gran medida los conocimientos e investigaciones derivadas de instituciones que como la UNAD están dispuestas a prestar diferentes servicios a través de sus programas como el de administración de empresas.
Los profesionales y estudiantes de este programa son privilegiados en las organizaciones del sector productivo, ocupando cargos directivos y estratégicos, porque los trabajos de investigación que se realizan mediante la realización de proyectos y experiencia profesional dirigida le permiten al estudiante obtener la experticia para enfrentarse al mundo laboral. Los egresados de administración de empresas se destacan en el ámbito laboral e investigativo, haciendo aportes relevantes a la sociedad, dejando en alto el nombre de la Universidad Nacional Abierta y Distancia - UNAD.

Se tendrá mejor cualificación de docentes, ya que no sólo se debe enfrentar a nuevas maneras de transmitir conocimientos sino a la carrera que impone lo instantáneo en el tiempo, la agilidad, la dedicación a la relación virtual con estudiantes virtuales; no necesariamente se necesitará cambiar el personal de docentes, sino se tiene que cambiar la metodología de aprendizaje, pues se requieren espacios donde se realice un proceso de contextualización, interacción, reflexión y diálogo para la construcción cooperativa de conocimiento, que permitirá valora el papel activo del tutor, como agente transformador de la universidad.

La innovación tecnológica y pedagógica, permitirá la consecución de material didáctico acorde con los temas a tratar y práctico que depende de la preparación de los docentes, siempre y cuando el material curricular pueda ser susceptible de ser actualizado constantemente y ajustado en cada aula virtual de conformidad con los requerimientos y las necesidades del mercado laboral.

La articulación de la investigación a la proyección comunitaria, el desarrollo regional e internacional, permitirán generar mayor conocimiento, gracias a la práctica obtenida en la aplicación del método científico, pues esta es una parte fundamental para descubrir las falencias y las 
posibles oportunidades que se presenten en todos los campos de aplicación, donde se le dé el tiempo suficiente a los docentes para realizar investigación aplicada para identificar las innovación tecnológica y pedagógicas que se presentarán en pro de con que permiten anticiparnos a lo que se requiere hacer desde ahora para contribuir a una excelente calidad de la educación en el programa de administración de empresas de la UNAD en el año 2019.

Por último si se logra la profesionalización y capacitación para los docentes y tutores; si se incentiva la innovación tecnológica y pedagógica y se articula la investigación a la proyección comunitaria, el desarrollo regional e internacional, se podrá elevar la calidad de la educación ofreciendo programas acordes con los requerimientos del mercado laboral y además se podrá realizar intercambio de experiencias con otras universidades involucrando las empresas y el estado, lo cual contribuirá para que el programa de administración de empresas de la UNAD sea pertinente y de vanguardia en el año 2019.

\section{Plan Prospectivo y Estratégico - Diseño de Estratégica}

Para la formulación de las estrategias se tiene en cuenta los cuatro pilares que la Comisión de la UNESCO ha señalado e ilustrado como bases de la Educación, y, que pueden ser un punto importante para comenzar: Aprender a conocer, aprender a hacer, aprender a vivir juntos y aprender a ser.

\section{Estrategia 1: Excelencia Docente}

Objetivo Estratégico: Lograr la cualificación de los docentes o tutores con especializaciones, maestrías y doctorados, para que de esta manera se contribuya para alcanzar la excelencia en la educación del programa de administración de empresas de la UNAD.

\section{Estrategia 2. Incentivar la Innovación, la Tecnología e Innovación tecno - pedagógica.}

Objetivo Estratégico: Fundamentar el desarrollo de la Escuela en la calidad de la docencia y de la investigación científica y aplicada, en estrategias de nuevas tecnologías de la comunicación y la información que complementen los Programas de Estudio y proyectos especiales, que permitan acreditar los programas de estudios presentes y futuros.

\section{Estrategia 3: Estimular la Investigación y el desarrollo tecnológico}

Objetivo Estratégico: Elaborar un estado del conocimiento sobre la educación a distancia y el e-learning que permita ubicar el trabajo de investigación dentro de las prioridades de la UNAD.

\section{Estrategia 4: Acreditación de alta calidad del programa de administración de empresas.}

Objetivo Estratégico: Crear sistemas de garantía de calidad de la educación en el programa de administración de empresas, donde se analice su desarrollo y resultado y se permita definir e implantar acciones de mejora continua de la calidad, con la participación de todos los implicados.

\section{Estrategia 5: Internacionalización}

Objetivo Estratégico: Buscar, avalar y concretar alianzas estratégicas con distintas entidades privadas, publicas, fundaciones, Universidades y ONGS a nivel Nacional e internacional para que el egresado de administración de empresas de la escuela de ciencias administrativas económicas y de negocios de la UNAD, tenga un papel más protagónico dentro del ámbito laboral, político, impacto social y económico en las distintas regiones del mismo y que generen, impulsen e inclusive activen el desarrollo del país en su totalidad; todo esto haciendo uso de la Investigación como plataforma y soporte para obtención de diagnósticos y de la toma de decisiones. 


\section{Discusión}

Para dar respuesta a la pregunta de ¿Qué estrategias se deben diseñar para construir el escenario apuesta del programa de administración de empresas de la UNAD, que permita que el egresado de nuestra Universidad sea de alta calidad, competitivo y reconocido en el mercado laboral nacional e internacional?

El estudio arrojo como resultado el diseño de 5 estrategias que permiten que el programa de administración de empresas de la Universidad Nacional abierta y a distancia UNAD, llegue al escenario apuesta denominado «Programa Integral».

Para ello se verifican las variables y su dependencia e influencia para el desarrollo de las estrategias que incluyen la formación docente, la innovación tecno pedagógica, la investigación y desarrollo tecnológico, Acreditación de alta calidad y la internacionalización como aspectos claves para el diseño de las estrategias. A continuación se relaciona las 5 estrategias diseñadas y su puesta en marcha.

\section{Estrategia 1: Excelencia Docente}

Esta estrategia se implementó de forma creciente en el programa, mediante el desarrollo de 2 actividades a) mejorar los perfiles de vinculación del cuerpo académico y b) favorecer la cualificación pos gradual del Cuerpo académico mediante la implementación del plan de desarrollo profesoral

\section{Estrategia 2. Incentivar la Innovación, la Tecnología e Innovación tecno - pedagógica.}

Esta estrategia inicio su implementación con el desarrollo de nuevos ambientes virtuales de aprendizaje, que los hace más interactivos y didáctico para el proceso de aprendizaje.

\section{Estrategia 3: Estimular la Investigación y el desarrollo tecnológico}

En esta estrategia se inició la identificación de los puntos fuertes en investigación de la escue- la y por ende del programa concentrando las líneas de investigación y los grupos que están adscritos a las líneas

\section{Estrategia 4: Acreditación de alta calidad del programa de administración de empresas.}

Esta estrategia se desarrolló y se obtuvo la acreditación de alta calidad otorgada por el Ministerio de Educación Nacional en marzo de 2012

\section{Estrategia 5: Internacionalización}

Esta estrategia se implementa mediante la operativizacion de los convenios de movilidad de la comunidad académica con diferentes instituciones de educación superior, centros de investigación y organismos internacionales.

En la actualidad el programa de Administración de empresas cuenta con acreditación de alta calidad otorgado por el Ministerios de Educación Nacional, Consolido sus líneas de investigación y ha entrado en proceso de Innovación curricular mediante núcleos problémicos para lograr llegar al escenario apuesta denominado «programa Integral».

Se encuentra en proceso de desarrollo de la estrategia de Excelencia Docente con la puesta en marcha del plan de desarrollo profesoral, y pendiente la Implementación las estrategia de Internacionalización e Innovación tecno pedagógica.

\section{Conclusiones}

El método de la prospectiva estratégica fue utilizado como una forma alternativa para generar una visión del Programa de administración de empresas de la UNAD al 2019.

Por medio del Método MIC-MAC, se identificaron trece (13) variables clave, que fueron seleccionadas, de treinta que dieron los expertos, 
así: Programa Certificado Con Alta Calidad, Perfil Del Cuerpo Académico, Acreditación De Alta Calidad, Cobertura A Nivel Nacional E Internacional, Acompañamiento tutorial, Sistemas De Comunicación, competencia, Aulas virtuales, Impacto Social, TIC, Sistemas de calidad, Mapas de conocimiento, Inversión En I \& D En Otras Instituciones Educativas

Las 5 hipótesis formuladas para la construcción de escenarios son el resultado de las variables clave que arroja el Método Mic Mac, las cuales son: ¿Articular la investigación a la proyección comunitaria, el desarrollo regional e internacional para que se genere mayor conocimiento; Incentivar la innovación tecnológica y pedagógica para un mejor desempeño teórico práctico; realizar intercambio de experiencias con otras universidades involucrando las empresas y el estado; elevar la calidad de la educación ofreciendo programas acordes con los requerimientos del mercado laboral y por último lograr la profesionalización y capacitación para los docentes y tutores contribuirá para que le programa de administración de empresas de la UNAD sea de excelente calidad en el año 2019?

El Método Sistema de Matrices de Impactos Cruzados (SMIC) permitió identificar El escenario apuesta Programa Integral que se da si se logra la profesionalización y capacitación para los docentes y tutores; si se incentiva la innovación tecnológica y pedagógica y se articula la investigación a la proyección comunitaria, el desarrollo regional e internacional, porque de esta manera se podrá elevar la calidad de la educación ofreciendo programas acordes con los requerimientos del mercado laboral y además se podrá realizar intercambio de experiencias con otras universidades involucrando las empresas y el estado, lo cual contribuirá para que le programa de administración de empresas de la UNAD sea de excelente calidad en el año 2019, logrando su acreditación.
Si se desarrolla la implementación de las 5 estrategias formuladas se lograra el escenario apuesta "Escenario Techo», que se orientan a los estándares de calidad del DEBER SER, hacia un ideal de excelencia, que pueden mostrar resultados específicos, tradición consolidada e impacto y reconocimiento social del programa de administración de Empresas.

\section{Referencias}

Balbi, Eduardo. (2008) Metodología de la Investigación de futuros: CelGyP y The Millenum Project, Buenos Aires - Argentina.

Berger. Gastón, (1957). Sciences humaines et previsión, Revue des deux mondes, № 1. París, Francia.

Berger, Gastón. (1967). Etapes de la prospective, Paris: PUF.

Godet. Michel, (1990). Prospektiker; Futuribles. Problemas y Métodos de Prospectiva: Caja de Herramientas. UNESCO.

Godet, Michel. \& Prospeckktiker (2000). La caja de herramientas de la prospectiva estratégica, Paris: LIPSOR - CNAM.

Godet, Michel. (2000). Manuel de Prospective Estratégique. Tome 2 L'art et la méthode. Paris.

Jouvenel. Hugues, (1993),.Sur la méthode prospective: un bref guide méthodologique,

Futuribles, № 179. Paris, Francia.

Mera, Carlos. (2006). Modulo de Prospectiva Estratégica. Bogotá: Universidad Nacional Abierta y a Distancia - UNAD.

Mojica, José. (2005). La Construcción de Futuro. Bogotá: Editorial Universidad Externado de Colombia / Convenio Andrés Bello.

Mojica, José. (2008). Dos modelos de la escuela voluntarista de prospectiva estratégica. Bogotá: Universidad Externado de Colombia.

Schwartz, Peter. (1991). The art of the long view., New York: Doubleday, 